\title{
Control of Electrically Excited Synchronous Motors with a Low Switching Frequency
}

\author{
Qing-qing $\mathrm{Yuan}^{\dagger}$, Xiao-jie $\mathrm{Wu}^{*}$, Peng Dai ${ }^{*}$, and Xiao Fu* \\ ${ }^{\dagger *}$ Dept. of Information and Electrical Eng., China University of Mining and Technology, Xuzhou, China
}

\begin{abstract}
The switching frequency of the power electronic devices used in large synchronous motor drives is usually kept low (less than 1 $\mathrm{kHz}$ ) to reduce the switching losses and to improve the converter power capability. However, this results in a couple of problems, e.g. an increase in the harmonic components of the stator current, and an undesired cross-coupling between the magnetization current component $\left(i_{m}\right)$ and the torque component $\left(i_{t}\right)$. In this paper, a novel complex matrix model of electrically excited synchronous motors (EESM) was established with a new control scheme for coping with the low switching frequency issues. First, a hybrid observer was proposed to identify the instantaneous fundamental component of the stator current, which results in an obvious reduction of both the total harmonic distortion (THD) and the low order harmonics. Then, a novel complex current controller was designed to realize the decoupling between $i_{m}$ and $i_{t}$. Simulation and experimental results verify the effectiveness of this novel control system for EESM drives.
\end{abstract}

Key words: Complex matrix model, Electrically excited synchronous motors (EESM), Hybrid observer, Complex current controller, Low switching frequency

\section{INTRODUCTION}

Electrically excited synchronous motors (EESM) are widely applied in high power industrial fields, e.g. metal rolling, mine hoisting, ship propulsion and locomotive traction, owing to their advantages such as a high efficiency, as well as a high and adjustable power factor [1]-[3]. The switching frequency of the power semiconductors is limited to below $1 \mathrm{kHz}$ to reduce the switching losses and to improve the output power of the converters [4]-[9]. However, a low switching frequency affects the performance of the control system. The harmonic components of the stator current increase with a reduction of the switching frequency, which affects normal operation [10]. In addition, a low switching frequency also causes a longer delay of the pulse width modulation (PWM), which deteriorates the dynamics of the current control and causes an undesired cross-coupling between $i_{m}$ and $i_{t}$ [11].

\footnotetext{
Manuscript received Nov. 29, 2011; revised May 3, 2012

Recommended for publication by Associate Editor Kyeong-Hwa Kim.

${ }^{\dagger}$ Corresponding Author: cumtxz1215@163.com

Tel: +86-13655205844, Fax: +86-0516-83885667, China Univ. of Mining and Tech

*Dept. of Information and Electrical Eng., China Univ. of Mining and Tech., China
}

For the vector control system, a fast torque response requires the fundamental component of the stator current, or the flux, to be successfully extracted and used as a feedback signal [10]. Extracting the fundamental content with filters introduces a signal delay and thus degrades the performance [10]-[12]. An open-loop fundamental observer was put forward in [13]. However, it didn't offer a correction link, which would result in a phase difference. A current fundamental observer was proposed for asynchronous machine drives, which provided a reference for EESMs [10]. Because of the model complexity of EESMs, some special issues need to be considered [6].

A number of solutions have been considered to deal with the decoupling problem of ac motors operating with a low switching frequency, such as pairs-angle matrix decoupling, unit-matrix decoupling, feedback control decoupling, voltage feed-forward decoupling, etc. [14]-[17]. However, all of these solutions are feasible only when the given current is equal to the real current, and the model parameters are matching [1], [18], [19]. In [20], a deadbeat control combined with a repetitive control was proposed to solve the PWM delay introduced by a low switching frequency. However, it did not provide a definite modeling [20].

There are two methods to establish the drive model, namely the classic matrix state space representation and the 
complex vector method. When compared with the classic matrix modeling, the complex vector method can reveal the inner electromagnetic relation [21]. Considering the rotor asymmetry of an EESM, a novel complex matrix modeling based on a combination of the matrix and complex vector methods is proposed, and it is convenient to design the control system [10], [21]-[23].

This paper presents a control system for an EESM with a low switching frequency based on the complex matrix modeling. A novel hybrid observer for identifying the instantaneous fundamental component of the stator current was proposed with a deep dynamic and steady state analysis. Furthermore, a novel current controller with complex state variables was designed to address the delay introduced by the switching device and the time-discrete sampling to realize the decoupling between $i_{m}$ and $i_{t}$, as well as the dynamic and steady response. The simulation and experimental results verify the proposed control scheme.

\section{COMPLEX MATRIX MODEL OF AN EESM}

Considering the rotor asymmetry of an EESM, the complex matrix models based on the rotor field coordinates can be expressed as in (1) (the damping windings were not included):

$$
\begin{aligned}
& \boldsymbol{u}_{s}=R_{s} i_{s}+\frac{d \psi_{s}}{d t}+j \omega_{r} \psi_{s} \\
& \boldsymbol{u}_{f}=R_{f} i_{f}+\frac{d \psi_{f}}{d t} \\
& \psi_{s}=l_{s} i_{s}+l_{m} i_{f} \\
& \psi_{f}=l_{m} i_{s}+L_{f} i_{f}
\end{aligned}
$$

where $\boldsymbol{u}_{s}$ and $\boldsymbol{u}_{f}$ are the voltages of the stator and the rotor field; $R_{s}$ and $R_{f}$ are the stator and field winding resistances; $\boldsymbol{\psi}_{s}$ and $\boldsymbol{\psi}_{f}$ are the flux linkage of the stator and the field; $\omega_{r}$ is the mechanical angular velocity of the rotor, which is equal to the angular velocity of the field coordinates; $\boldsymbol{I}_{s}=\left[\begin{array}{cc}L_{d} & 0 \\ 0 & L_{q}\end{array}\right]$ is the inductance coefficient of the stator; $\boldsymbol{I}_{m}=\left[\begin{array}{cc}L_{m d} & 0 \\ 0 & L_{m q}\end{array}\right]$ is the mutual inductance between the stator and the rotor; $L_{f}$ is the inductance coefficient of the rotor; $L_{d}$ and $L_{q}$ are the synchronous inductance coefficients; and $L_{m d}$ and $L_{m q}$ are the armature reaction coefficients.

By using the Laplace transformation, the motor model in (1) can be transferred as (2):

$$
\begin{aligned}
& \boldsymbol{\tau}_{s}^{\prime} \frac{d \psi_{s}}{d t}+\psi_{s}=-j \omega_{r} \boldsymbol{\tau}_{s}^{\prime} \psi_{s}+\boldsymbol{k}_{f} \psi_{f}+\tau_{s}^{\prime} \boldsymbol{u}_{s} \\
& \boldsymbol{\tau}_{f}^{\prime} \frac{d \psi_{f}}{d t}+\psi_{f}=\boldsymbol{k}_{s} \psi_{s}+\tau_{f}^{\prime} \boldsymbol{u}_{f}
\end{aligned}
$$

where $\boldsymbol{\sigma}=1-\boldsymbol{I}_{m}{ }^{2} /\left(\boldsymbol{I}_{s} L_{f}\right)$ is the total leakage factor; $\boldsymbol{\tau}_{s}{ }^{\prime}=\boldsymbol{\sigma} \boldsymbol{l}_{s} / \boldsymbol{R}_{s}$ is the transient time constant of the stator; $\boldsymbol{\tau}_{f}{ }^{\prime}=\sigma L_{f} / R_{f}$ is the transient time constant of the rotor; and $\boldsymbol{k}_{f}=\boldsymbol{I}_{m} / L_{f}$ and $\boldsymbol{k}_{s}=\boldsymbol{I}_{m} / \boldsymbol{I}_{s}$

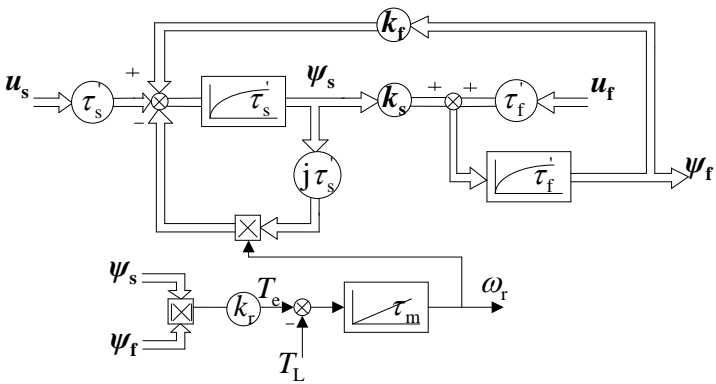

Fig. 1. Complex matrix model of an electrically excited motor.

are the coupling factor of the rotor and the stator. Equation (2) can be visualized in the signal flow chart (see Fig. 1), where $\tau_{m}$ is the mechanical time constant; $T_{e}$ and $T_{L}$ are the electromagnetic and load torque respectively; and $k_{r}$ is a calculation constant.

\section{HYBRID CURRENT OBSERVER}

\section{A. Design of the hybrid observer}

As mentioned in the introduction, the fast control of torque requires the fundamental component of the stator current in the vector control system. Considering the effect of a low switching frequency, a novel hybrid observer was proposed to extract the fundamental current of the stator. A complex matrix model for an EESM was established in a hybrid structure as a combination of the stator model in the stationary coordinates and the rotor model in the field coordinates

$$
\begin{aligned}
& \boldsymbol{u}_{s}^{(S)}=R_{s} i_{s}^{(S)}+p \psi_{s}^{(S)} \\
& \boldsymbol{i}_{s}^{(S)}=\left(\psi_{s}^{(S)}-\boldsymbol{k}_{r} \psi_{f}^{(S)}\right) / \boldsymbol{\sigma} \boldsymbol{l}_{s} \\
& \boldsymbol{u}_{f}^{(F)}=R_{f} i_{f}^{(F)}+p \psi_{f}^{(F)} \\
& \psi_{f}^{(F)}=L_{m d} i_{s d}+L_{f} i_{f}^{(F)}
\end{aligned}
$$

where the superscripts $(S)$ and $(F)$ represent the stationary coordinates and the field coordinates; and $i_{s d}$ is the direct-axis component of the stator current. Transforming (3) to (4) yields the following transfer function:

$$
\begin{aligned}
& \boldsymbol{\tau}_{S}^{\prime} \frac{d \boldsymbol{\psi}_{S}^{(S)}}{d t}+\psi_{S}^{(S)}=\boldsymbol{\tau}_{S}^{\prime} \boldsymbol{u}_{S}^{(S)}+\boldsymbol{k}_{r} \psi_{f}^{(S)} \\
& \tau_{f} \frac{d \boldsymbol{\psi}_{f}^{(F)}}{d t}+\psi_{f}^{(F)}=L_{m d} i_{s d}+\tau_{f} \boldsymbol{u}_{f}^{(F)}
\end{aligned}
$$

where, $\tau_{f}=L_{f} / R_{f}$ is the time constant of the rotor.

The principle of the hybrid observer is to take the machine model as the observer model, then adopt an undistorted reference voltage vector $\boldsymbol{u}_{s}{ }^{*}$ as the input of the observer which replaces the real voltage input $\boldsymbol{u}_{\mathrm{s}}$, as shown in Fig.1. A new stator flux and current is calculated by the actual signals such as the stator current, the speed information and the estimated stator flux. Since there are no harmonic components in the main input signal of this observer, the 


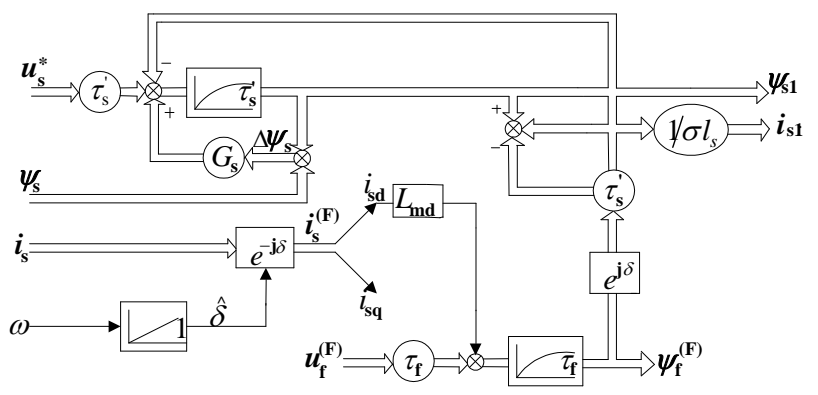

Fig. 2. Structure of the hybrid observer for the stator observer.

calculated current or flux could be regarded as the fundamental component of the actual signal. The equations of the fundamental observer are as follows:

$$
\begin{aligned}
& \boldsymbol{\tau}_{S}^{\prime} \frac{{\hat{d \psi_{s 1}}}_{d t}^{(S)}+\hat{\psi}_{S 1}^{(S)}=\boldsymbol{\tau}_{S}^{\prime} u_{S}^{*}+\boldsymbol{k}_{f} \boldsymbol{\psi}_{f}^{(S)}+G_{S} \Delta \boldsymbol{\psi}_{S}^{(S)}}{\tau_{f} \frac{d \boldsymbol{\psi}_{f}^{(F)}}{d t}+\psi_{f}^{(F)}=L_{m d} i_{s d}+\tau_{\mathbf{f}} \boldsymbol{u}_{f}^{(F)}} \\
& \hat{i}_{S 1}^{(S)}=\frac{\hat{\psi}_{s 1}^{(S)}-\boldsymbol{k}_{f} \boldsymbol{\psi}_{f}^{(S)}}{\boldsymbol{\sigma} \boldsymbol{l}_{S}}
\end{aligned}
$$

$\hat{\boldsymbol{\psi}}_{s 1}^{(S)}$ and $\hat{\boldsymbol{i}}_{s 1}^{(S)}$ are observed fundamental

where, $\boldsymbol{\psi}_{s 1}$ and $\boldsymbol{i}_{s 1}$ are observed fundamental
components of the stator current and the flux, respectively; $\Delta \psi_{S}^{(S)}=\psi_{S}^{(S)}-\hat{\psi}_{S 1}^{(S)}$ is the estimated stator flux error, and $\boldsymbol{\Psi}_{s}^{(S)}$ is the actual stator flux calculated by the flux observer. $\boldsymbol{G}_{s}$ is the error correction signal, and it was chosen as $\boldsymbol{G}_{s}=g+j 0$. This selection is justified by the observer given in (5), which does not exhibit imaginary terms, where $g$ is an adjustable parameter and can be obtained by the pole-zero placement of the matrix. The structure of the hybrid observer for the stator current is shown in Fig.2.

\section{B. Stability analysis of the hybrid observer}

The impulse response of the error function was adopted to analyze the stability of the hybrid observer with variable speeds, adjustable parameters and motor parameter mismatches of the machine model. The error function of the observer is given in (6) from (4) and (5).

$$
\Delta \psi_{s}=\frac{\tau_{s}^{\prime}\left(\boldsymbol{u}_{s}^{*}-\boldsymbol{u}_{s}\right)}{\tau_{s}^{\prime} s+1+j \omega_{r} \tau_{s}^{\prime}+\boldsymbol{G}_{s}}
$$

The impulse response of (6) is shown in Fig.3.

It can be seen that variations in the speed have little influence on the error impulse response, as in Fig.3 (a), where the speed varies from $0.5 \omega_{n}$ to $\omega_{n}$ ( $\omega_{n}$ is the rated speed). Fig.3 (b) shows a function of the adjustable parameter $g$, which indicates that large $g$ leads to better performance. When the system operates at its rated speed and a parameter error set to $25 \%$ of the actual value, the error impulse

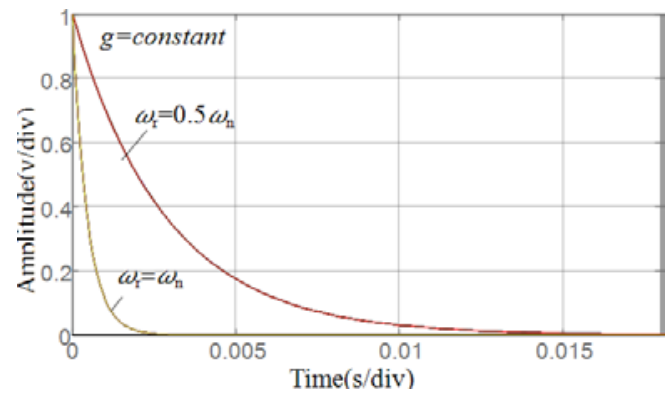

(a) Impulse response at different speed.

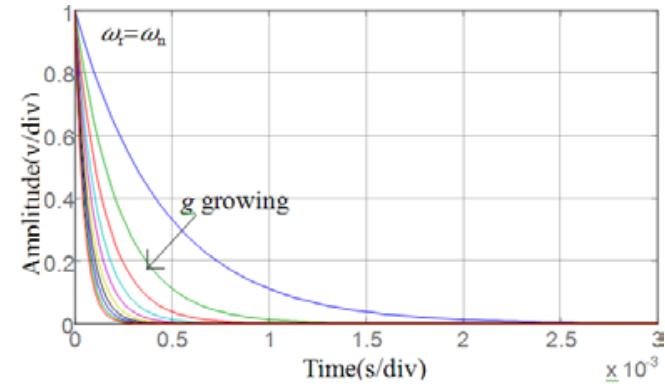

(b) Impulse response at different adjustable parameter.

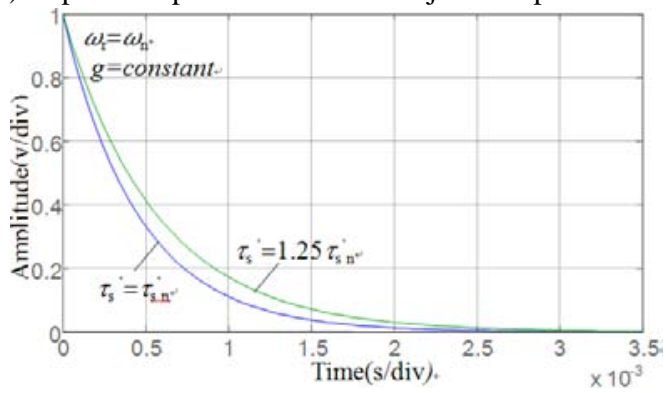

(c) Impulse response with a parameters' mismatch.

Fig. 3. Impulse response of observed error function.

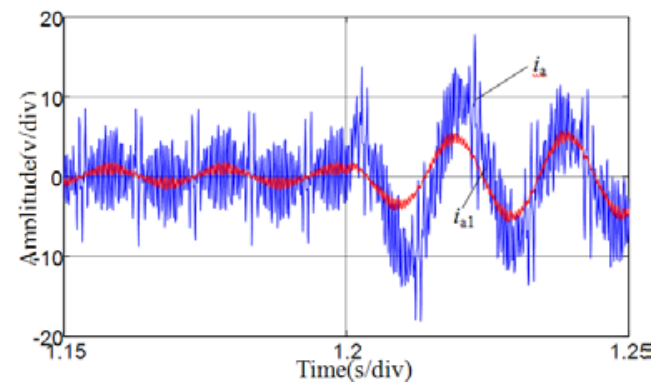

(a) $f_{\mathrm{s}}=1 \mathrm{kHz}$.

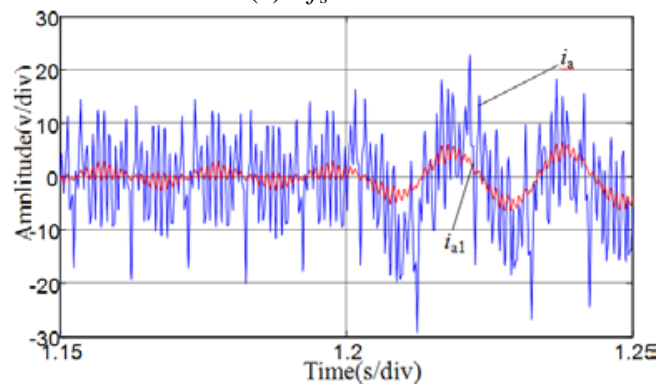

(b) $f_{\mathrm{s}}=500 \mathrm{~Hz}$.

Fig. 4. Dynamic response of the observed stator current at different switching frequency. 


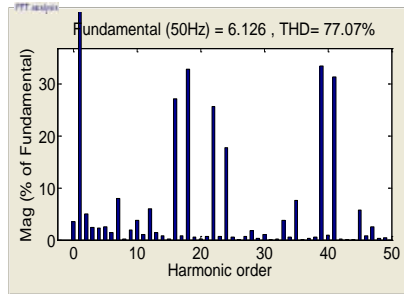

(a) Actual stator current.

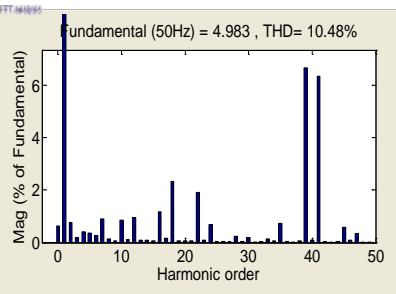

(b) Observed stator current.
Fig. 5. FFT analysis of the stator current at $f_{\mathrm{s}}=1 \mathrm{kHz}$.

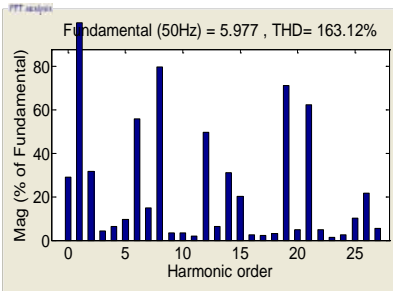

(a) Actual stator current.

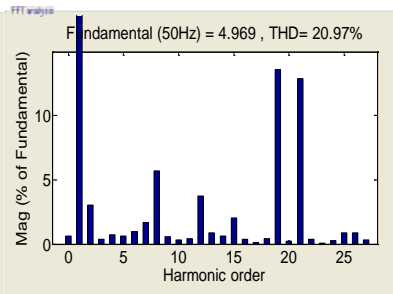

(b) Observed stator current.
Fig. 6. FFT analysis of the stator current at $f_{\mathrm{s}}=500 \mathrm{~Hz}$.

response is shown in Fig.3 (c). This demonstrates the strong robustness of the observer.

\section{Simulation and harmonic analysis}

Simulations were carried out for a two-level EESM with different switching frequencies $(1 \mathrm{kHz}, 500 \mathrm{~Hz})$ using the MATLAB tool. The results are shown in Fig. 4.

As can be seen, this kind of hybrid observer can extract the fundamental current component with a dynamic characteristic and a steady accuracy. In addition, the dynamic response time was less than one $T_{\mathrm{s}}$ (switching period). For a further study, an FFT analysis was carried out on the actual and observed stator current, as shown in Fig. 5 and Fig. 6.

In [24], it was pointed out that the frequency spectrum of an inverter (two-level) can be decomposed into three parts: (1) the target fundamental component; (2) the low order harmonics, which are the main factor affecting performance; (3) the carrier harmonic components and their sidebands, usually considered as high order harmonics. For a three phase inverter, the frequency of part (3) can be represented as $f_{s} \pm 2 f_{0}$, $f_{\mathrm{s}} \pm 4 f_{0}, 2 f_{\mathrm{s}} \pm f_{0}, 2 f_{\mathrm{s}} \pm 5 f_{0}, 2 f_{\mathrm{s}} \pm 7 f_{0}$ ( $f_{\mathrm{s}}$ is the switching frequency and $f_{0}$ is the target fundamental frequency), and so on.

From Fig. 5 (a) and Fig. 6 (a), it can be seen that, when $f_{\mathrm{s}}=1 \mathrm{kHz}$ and $f_{0}=50 \mathrm{~Hz}$, the THD of the phase current is $77.07 \%$, and the harmonic orders of part (3) are 16, 18, 22, 24 and $33,35,39,41,45,47$, which could still be taken as high order harmonics. However, when $f_{\mathrm{s}}=500 \mathrm{~Hz}$ and $f_{0}=50 \mathrm{~Hz}$, the THD is as high as $163.12 \%$ and the harmonic orders are 6,8 , 12,14 and $13,15,19,21,25,27$, which are thought as relatively low order harmonics, with a severe effect on the performance of the system. The FFT analysis on the observed phase currents are shown in Fig. 5 (b) and Fig. 6 (b). It can be seen that there is a great reduction of the THD $(10.48 \%$ at $f_{\mathrm{s}}=1 \mathrm{kHz}, 20.97 \%$ at $f_{\mathrm{s}}=500 \mathrm{~Hz}$ ). This is especially true for the low order harmonic components.

\section{COMPLEX CURRENT CONTROLLER}

\section{A. Modeling of a current loop with a low switching frequency}

For the vector control system, a cross-coupling term exists between $i_{m}$ and $i_{t}$ as a result of (1) the cross-coupling identity of the control object itself; (2) the cross-coupling caused by a PWM delay with a low switching frequency. The proportional integral (PI) controller and the feed-forward compensation can only resolve the former cross-coupling. The transfer function of the current loop can be expressed as:

$$
F(s)=\frac{\boldsymbol{u}_{s}}{\boldsymbol{i}_{s}}=\frac{1 / R_{s}}{\tau_{s} s+1+\mathrm{j} \omega_{r} \tau_{s}}
$$

where $\tau_{s}=L_{s l} / R_{s}$. The delay introduced by the PWM inverter and the digital sampling are approximated by a first-order differential equation [25]:

$$
\tau_{d} \frac{\mathrm{d} \boldsymbol{u}_{s}}{\mathrm{~d} t}+\left(1+\mathrm{j} \omega_{r} \tau_{d}\right) \boldsymbol{u}_{\mathrm{s}}=\boldsymbol{u}^{*}
$$

where $\boldsymbol{u}^{*}$ is the reference voltage vector produced by the current controller, $\tau_{\mathrm{d}}=0.75 / f_{\mathrm{s}}$ is the time constant of the delay, and (8) can be transferred to:

$$
F_{d}(s)=\frac{1}{\left(\tau_{d} s+1+j \omega_{r} \tau_{d}\right)}
$$

The complex transfer function of the current loop is:

$$
F_{m i}(s)=\frac{1 / R_{S}}{\tau_{s} s+1+j \omega_{r} \tau_{s}} \cdot \frac{1}{\tau_{d} s+1+j \omega_{r} \tau_{d}}
$$

\section{B. Analysis of the low switching frequency}

Equation (10) can be represented as:

$$
F_{m i}(s)=\operatorname{Re}\left[F_{m i}(s)\right]+\operatorname{Im}\left[F_{m i}(s)\right]
$$

Cross-coupling is introduced by the complex factor $j$, which means that the imaginary component of a complex transfer function determines the cross-coupling effect of a dynamic system [25]. The definition of the frequency response of the cross-coupling is given as:

$$
F_{X Y}(\mathrm{j} \omega)=\frac{\operatorname{Im}\left[F_{X Y}(j \omega)\right]}{\operatorname{Re}\left[F_{X Y}(j \omega)\right]}
$$

With (12) applied to equation (10), the cross-coupling response of the current loop along with the angular frequency $\omega$ with a different switching frequency is shown in Fig.7.

\section{Complex current controller}

From the foregoing analysis, it can be seen that the decoupling between $i_{m}$ and $i_{t}$ can be realized when the current controller eliminates the complex pole existing in the control plant. $F_{r}(s)$ is the complex controller to be designed:

$$
F_{r}(s)=k_{0} \frac{\left(\tau_{s} s+1+j \omega_{r} \tau_{s}\right)\left(\tau_{d} s+1+j \omega_{r} \tau_{d}\right)}{\tau_{s} s\left(\tau_{d} s+1\right)}
$$

Then, the whole open loop transfer function is derived from (10) and (13): 


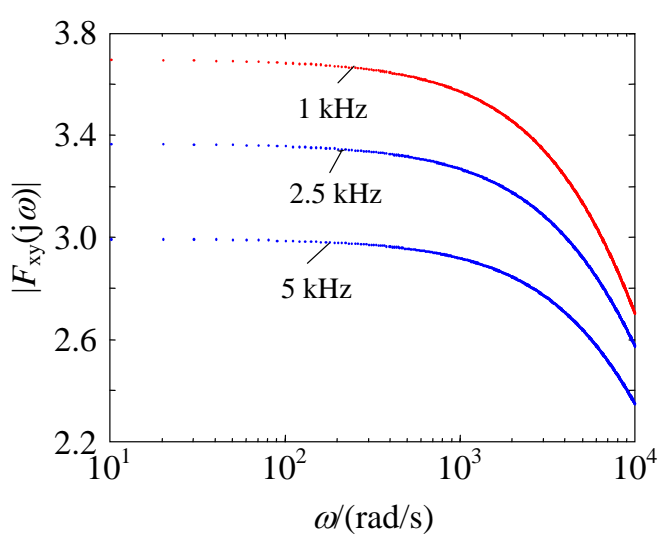

Fig. 7. Cross-coupling response of the current loop along with the angular frequency $\omega$ at different switching frequency.

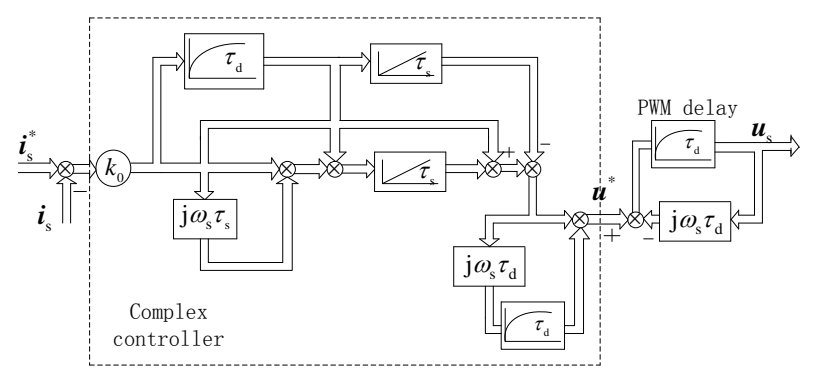

Fig. 8. Diagram of complex current regulator.

$$
\begin{aligned}
F_{C}(s)= & \frac{\boldsymbol{u}_{s}}{\boldsymbol{i}_{s}}=F(s) \times F_{d}(s) \times F_{r}(s) \\
= & \frac{1 / R_{s}}{\tau_{s} s+1+\mathrm{j} \omega_{r} \tau_{s}} \times \frac{1}{\left(\tau_{d} s+1+\mathrm{j} \omega_{r} \tau_{d}\right)} \times \\
& k_{0} \frac{\left(\tau_{s} s+1+\mathrm{j} \omega_{r} \tau_{s}\right)\left(\tau_{d} s+1+\mathrm{j} \omega_{r} \tau_{d}\right)}{\tau_{s} s\left(\tau_{d} s+1\right)} \\
= & \frac{k_{0} / R_{s}}{\tau_{s} s\left(\tau_{d} s+1\right)}
\end{aligned}
$$

With all of the complex poles being eliminated, the open loop transfer function is resulted to (14), which no longer has a more complex coefficient. As a result, the cross-coupling is eliminated. A signal diagram of the complex current regulator is shown in Fig.8, in which, $k_{0}$ is the adjustment coefficient related to the gain of the open loop.

\section{Simulation analysis}

Simulations of the complex current controller were carried out with different switching frequencies $(1 \mathrm{kHz}$ and $500 \mathrm{~Hz})$ after obtaining the fundamental current component. The aim of these simulations was to analyze the cross-coupling between $i_{m}$ and $i_{t}$, which was shown in Fig. 9 and Fig. 10. There is no-load before Time $=1 \mathrm{~s}$, and the given speed was $20 \mathrm{rad} / \mathrm{min}$ at Time $=0.2 \mathrm{~s}$ and $1500 \mathrm{rad} / \mathrm{min} \quad\left(\omega_{r}=\omega_{n}\right)$ at Time $=0.4 \mathrm{~s}$. Half of the load was added at Time $=1 \mathrm{~s}$.

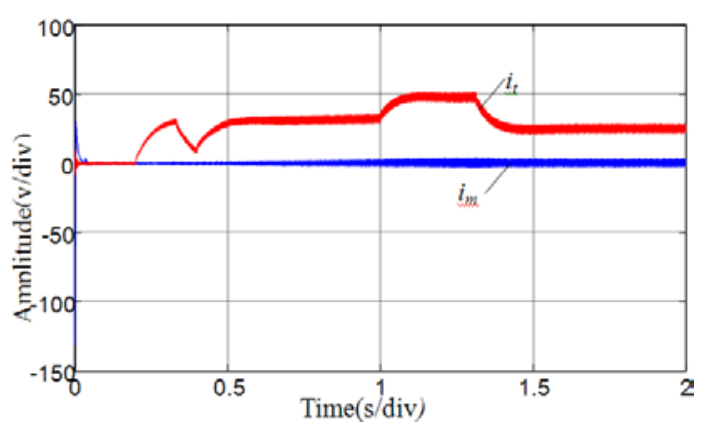

Fig. 9. Current waveforms of $M T$ coordinates at $f_{\mathrm{s}}=1 \mathrm{kHz}$, complex controller.

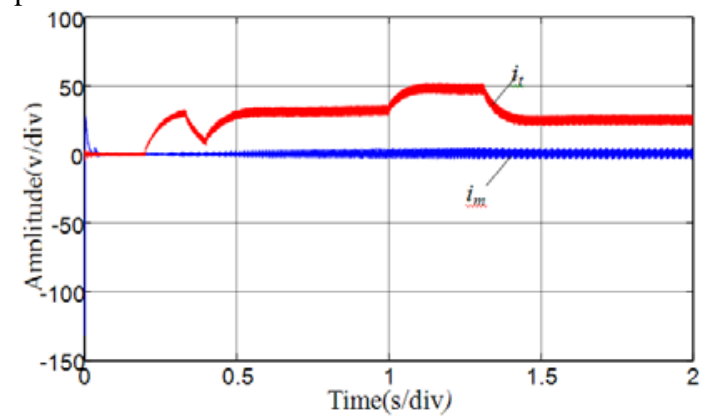

Fig.10. Current waveforms of $M T$ coordinates at $f_{\mathrm{s}}=500 \mathrm{~Hz}$, complex controller.

This kind of current controller, with its complex state variables, can realize the decoupling of $i_{m}$ and $i_{t}$ as well as fast dynamic and steady responses.

\section{EXPERIMENTAL RESULTS}

The whole control system takes the fundamental current component generated by the hybrid observer, as a feedback signal, which is shown in Fig.11. This system adopts the air gap flux linkage orientation.

The experimental results were obtained from a $2.5 \mathrm{kVA}$ EESM fed by a two-level inverter and a load from a dc motor. The motor parameters are listed in the APPENDIX.

The first test was the closed-loop control of the EESM at different switching frequencies $(5 \mathrm{kHz}, 500 \mathrm{~Hz})$. The time-discrete control signals were processed at $10 \mathrm{kHz}$. Then experiments were conducted on the hybrid observer and the complex current regulator.

Fig.12 shows the extraction of the fundamental current component of the a-phase at the steady-state. Although the switching frequency was as low as $500 \mathrm{~Hz}$, an immediate response was obtained without errors with respect to the phase angle and the magnitude. The dynamic performance of the complex current regulator was shown in Fig.13. When increasing and reducing the load, there was a relatively small cross-coupling between $i_{m}$ and $i_{t}$. 


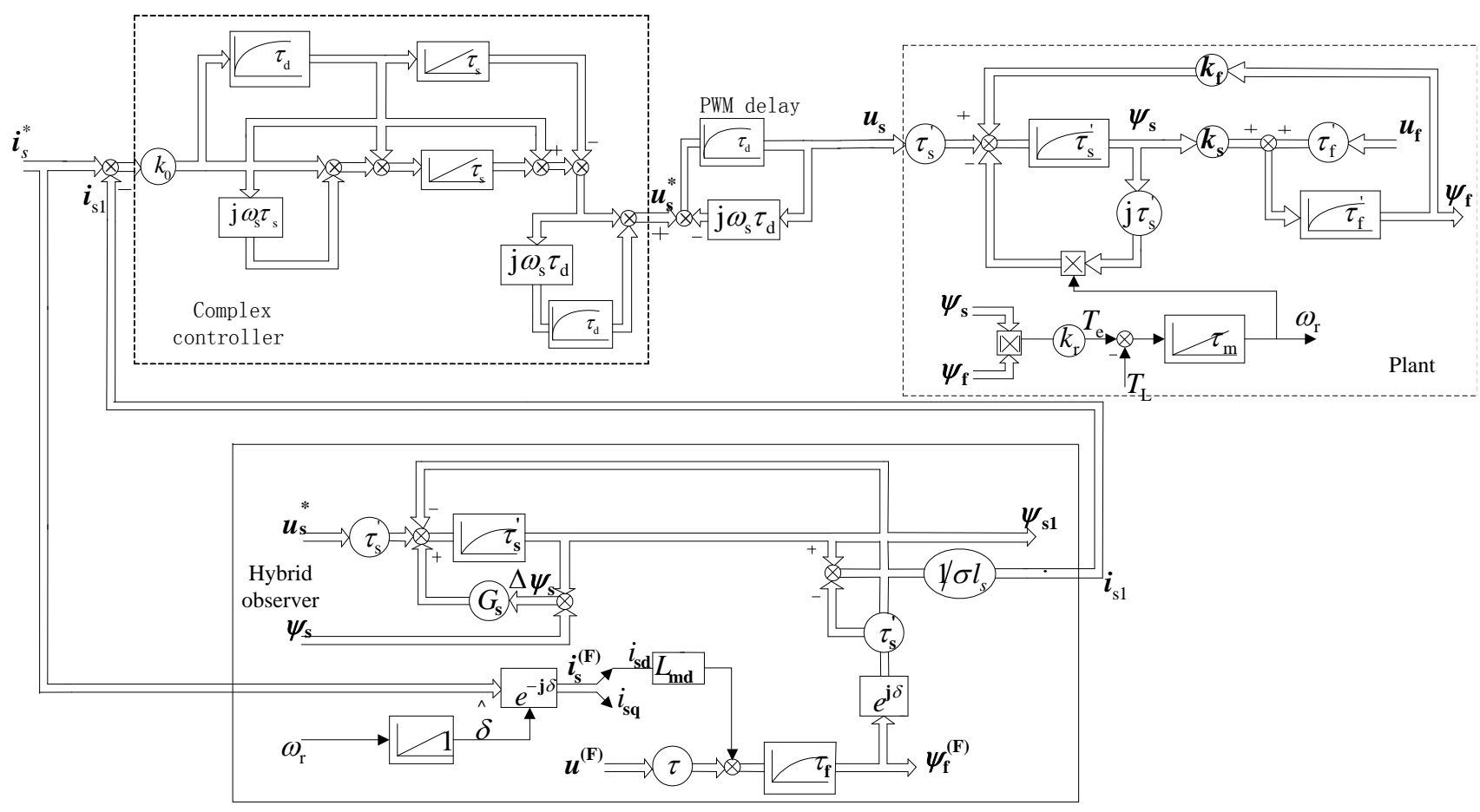

Fig.11. Diagram of the whole control system.

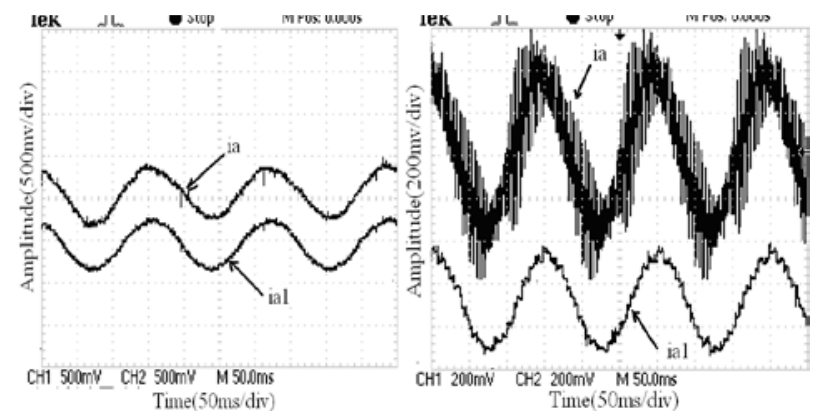

Fig. 12. Estimated fundamental component of the a-phase at different frequency (a) $f_{\mathrm{s}}=5 \mathrm{kHz}$; (b) $f_{\mathrm{s}}=500 \mathrm{~Hz}$.

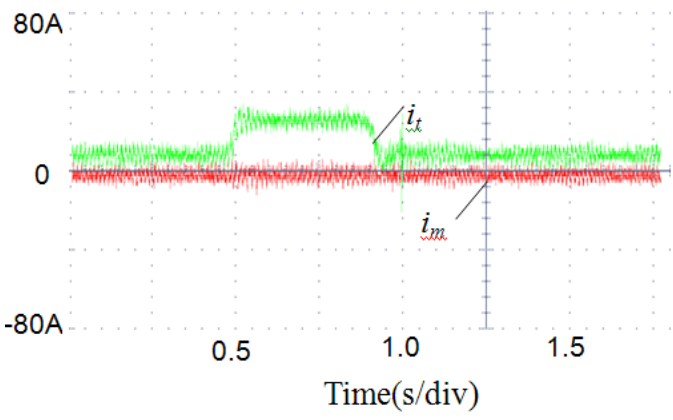

Fig. 13. current waveforms of $M T$ coordinates at $f_{\mathrm{s}}=500 \mathrm{~Hz}$ stability of ac machines, advanced control of electrical machines, and power electronics.

\section{CONCLUSION}

Considering the complexity of an EESM, a novel complex matrix model has been established by a combination of the classic matrix model and the complex model. This modeling of an electrically excited motor in terms of the complex matrix provides a better insight into the dynamics of the controlled system.

The harmonic current components increase along with the reduction of the switching frequency. The fast dynamic control requires an estimation of the instantaneous fundamental current as a feedback signal. One hybrid fundamental observer at different coordinates was proposed for this purpose. This can observe the fundamental components of the stator current in real-time at a switching frequency of only $500 \mathrm{~Hz}$ (fed by a two-level inverter).

According to the cross-coupling between $i_{m}$ and $i_{t}$ of a vector controlled drive, a novel complex current controller was established to realize the decoupling. Simulation and experimental results verify that this current controller with its complex state variables can realize the decoupling of $i_{m}$ and $i_{t}$ as well as perfect the dynamic and steady responses.

Furthermore, an improvement of the PWM modulation should be carried out to obtain a better dynamic performance of the control system for electrically excited drives with a low switching frequency.

\section{APPENDIX}

Machine data:

$R_{s}=6.08 \Omega, \quad R_{f}=16.47 \Omega, \quad L_{s l}=0.0358 \mathrm{H}, \quad L_{f l}=0.000623 \mathrm{H}$, 
$L_{m d}=0.322 \mathrm{H}, \quad L_{m q}=0.0148 \mathrm{H} ;$ Rated power $=2.5 \mathrm{kVA}$, Rated speed=1500rpm, Number of pole-pairs=2, Conversion coefficient $=0.08413$.

\section{ACKNOWLEDGMENT}

The authors would like to thank China National Natural Science Foundation (51077124) and the Fundamental Research Funds for the China Central Universities (2010QNB32) and the Research and Innovation Program of Postgraduates in Jiangsu Province (CXZZ12_0930)

\section{REFERENCES}

[1] D. Beliav, A. Weigner, R. Paes, and S. Weigel, "Field oriented control of a synchronous drive,” IEEE International Conference on Electric Machines and Drives, pp. 957-961. 2005.

[2] J. A. Pontt, J. R. Rodriguez, A. Liendo, P . Newman, and J. Holtz, "Network friendly low-switching-frequency multi-pulse high-power three-level PWM rectifier," IEEE Trans. Ind. Electron., Vol. 56, No. 4, pp. 1254-1262, Apr. 2009.

[3] M. Nemec, K. Drobnic, D. Nedeljkovic, and V. Ambrozic, "Direct current control of a synchronous machine in field coordinates," IEEE Trans. Ind. Electron., Vol. 56, No. 10, pp. 4052-4061, Oct. 2009.

[4] B. K. Bose, Power electronics and motor drives: advances and trends, Elsevier/ Academic Press, 2006.

[5] A.K. Rathore, J. Holtz, and T. Boller, "Synchronous optimal pulsewidth modulation for low switching frequency control of medium voltage multi-level inverters,” IEEE Transactions on Industrial Electronics, Vol. 57, No. 7, pp. 1419-1423, Jul. 2010.

[6] N. Oikonmou, Control of Medium-Voltage Drives at Very Llow Switching Frequency, Logos Verlag Berlin GmbH, Chap.2, 2008.

[7] L. G. Franquelo, J. I. Leon, and E. Dominguez, "New trends and topologies for high power industrial applications: The multilevel converters solution,” International Conference on Power Engineering Energy and Electrical Drives, pp. 1-6. 2009.

[8] X. L. Ma, "Stator flux trajectory tracking control synthesis of low switching frequency medium voltage drive," The World of Inverters, No.12, pp. 45-51, Dec. 2009.

[9] A. G. Siemens, "Power semiconductors: for medium voltage converters-an overview," 13th European Conference on Power Electronics and Applications, PP. 121-134. 2009.

[10] J. Holtz, N. Oikonomou, "Estimation of the fundamental current in low-switching-frequency high dynamic mediumvoltage drives," IEEE Trans. Ind. Appl., Vol. 44, No.5, pp. 1597-1605, Sep/Oct. 2008.

[11] G. F. Franklin, J. D. Powell, and A. E. Naeini, Feedback Control of Dynamic Systems, New York Prentice Hall, Chap. 2, 2009.

[12] J. Holtz and N. Oikonomou, "Fast dynamic control of medium voltage drives operating at very low switching frequency-an overview," IEEE Trans. Ind. Electron., Vol. 55, No.3, pp. 1005-1013, Jan/Feb. 2008.

[13] C. Silva and J. Oyarzun, "High dinamic control of a PWM rectifier using harmonic elimination,” 32th Annual Conference of the IEEE Industrial Electronics Society, pp. 2569-2574. 2006.

[14] Z. G. Zhou, "A induction motor de-coupling control method," Proceeding of the CSEE, Vol. 23, No. 2, pp. 121-125, Jan. 2003.

[15] J. Rodriguez, P. Cortes, R. Kennel, and M. P. Kazrnierkowski, "Model predictive control-a simple and powerful method to control power converters," Power Electronics and Motion Contro Conference, pp. 41-49, 2009.

[16] C. J. Li, Ac Synchronous Motor Speed Control System , China Science Press, Chap.5, 2006.

[17] H. Zhang, K.P. Liu, and M. Braun, "The algorithm of real-time solution of selective harmonic controlling equations for PWM with low switching frequency,” Proceedings of the CSEE, Vol. 26, No. 22, pp. 80-84, Aug. 2006.

[18] Y. S. Zhou and J. G. Jiang, "Dynamic decoupling control for asynchronous motor,” The Middle and Small Motor, No. 2, pp. 22-26, Feb. 2001.

[19] X. L. Ma, High-Power Cycloconverter-Fed Speed Control and Vector Control Technology, China Machine Press, Chap.5, 2003.

[20] J. Shih-liang, H. Hsiang-sung, and T. Ying-yu, “A three-phase PWM AC-DC converter with low switching frequency and high power factor using DSP-based repetitive control technique," 29th Annual IEEE Power Electronics Specialists Conference, pp. 517-523. 1998.

[21] J. Holtz, "The representation of AC machine dynamics by complex signal flow graphs," IEEE Trans. Ind. Electron., Vol. 42, No. 3, pp. 263-271, June. 1995.

[22] K. Dai, P. G. Liu, J. Xiong, and J. Chen, “Comparative study on current control for three- phase SVPWM voltagesource converter in synchronous rotating frame using complex vector method," The 3rd International Workshop 
on Compatibility in Power Electronics, pp. 695-700. 2003.

[23] A. R. Rezazade, A. Sayyah, and M. Aflaki, "Modulation error observation and regulation for use in off-line optimal PWM fed high power synchronous motors," $1^{\text {st }}$ IEEE Conference on Industrial Electronics and Applications, pp. 141-155. 2006.

[24] D. Grahame Holmes, Thomas A. Lipo, Pulse width modulation for power converters: principles and practice, Wiley Publishing, 2003.

[25] J. Holtz, J. Quan, G. Schmitttt, J. Pontt, J. Rodriguez, and P.Newman, H. Miranda, "Design of fast and robust current regulators for high-power drives based on complex state variables,” IEEE Trans. Ind. Applications, Vol. 40, No. 5, pp. 1388-1397, Sep/Oct. 2004.

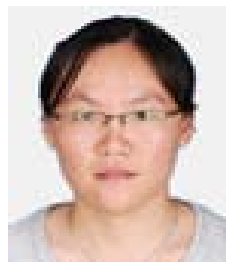

Qing-qing Yuan was born in NanTong JiangSu, China in 1987. She received her B.S. and M.S. from the China University of Mining and Technology, China, in 2009 and 2011, respectively. Since 2011, she has been working towards her Ph.D. in the Department of Information and Electrical Engineering, China University of Mining and Technology. Her current research interests include the modeling and control of high power drives with a low switching frequency.

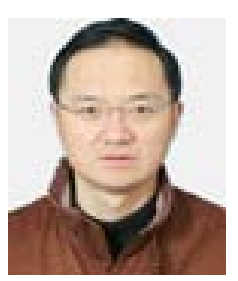

Xiao-jie Wu was born in HengYang, HuNan, China, in 1966. He received his B.S. in Industrial Automation from the China University of Mining and Technology, China, in 1988, and his M.S. and Ph.D. in Electrical Engineering from the China University of Mining and Technology, China, in 1991 and 2000, respectively. From 2002 to 2004, he did postdoctoral research at Tsinghua University, Beijing, China. Since 1991, he has been with the Department of Information and Electrical Engineering, China University of Mining and Technology, China, where he is currently a Professor. His current research interests include the stability of ac machines, advanced control of electrical machines, and power electronics.

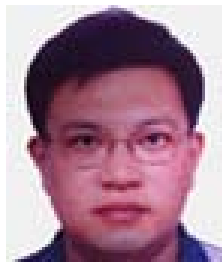

Peng Dai was born in HuaiBei, AnHui, China, in 1973. He received his B.S. in Electrical Engineering from the AnHui University of Science and Technology, Huainan, China, in 1994, and his M.S. and Ph.D. in Electrical Engineering from the China University of Mining and Technology, China, in 1998 and 2006, respectively. Since 1998, he has been with the Department of Information and Electrical Engineering, China University of Mining and Technology, China, where he is currently a Professor. His current research include the stability and control of synchronous machines.

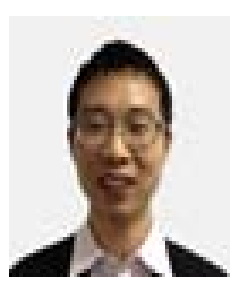

Automation, China
Xiao Fu was born in TaiAn, ShanDong, China, in 1985. He received his B.S., M.S. and Ph.D. in Electrical Engineering from the China University of Mining and Technology, China, in 2006, 2008 and 2011, respectively. Since 2012, he has been working for Advanced Technology, Rockwell 